\title{
DOLBEAULT HOMOTOPY THEORY AND COMPACT NILMANIFOLDS
}

\author{
L. A. CORDERO \\ Departamento de Geometría y Topología, Facultad de Matemáticas \\ Universidad de Santiago de Compostela, 15705 Santiago de Compostela, Spain \\ E-mail: cordero@zmat.usc.es \\ M. FERNÁNDEZ \\ Departamento de Matemáticas, Facultad de Ciencias \\ Universidad del País Vasco, Apartado 644, 48080 Bilbao, Spain \\ E-mail: mtpferol@lg.ehu.es \\ A. GRAY \\ Department of Mathematics, University of Maryland \\ College Park, Maryland 20742, U.S.A. \\ E-mail: gray@bianchi.umd.edu \\ L. UGARTE \\ Departamento de Matemáticas (Geometría y Topología) \\ Facultad de Ciencias, Universidad de Zaragoza \\ Campus Plaza San Francisco, 50009 Zaragoza, Spain \\ E-mail: ugarte@posta.unizar.es
}

\begin{abstract}
In this paper we study the degeneration of both the cohomology and the cohomotopy Frölicher spectral sequences in a special class of complex manifolds, namely the class of compact nilmanifolds endowed with a nilpotent complex structure. Whereas the cohomotopy spectral sequence is always degenerate for such a manifold, there exist many nilpotent complex structures on compact nilmanifolds for which the classical Frölicher spectral sequence does not collapse even at the second term.
\end{abstract}

1. Introduction. For a compact nilmanifold (i.e. a homogeneous space of the form $\Gamma \backslash G$, where $G$ is a simply-connected nilpotent Lie group and $\Gamma$ a lattice of $G$ of maximal rank) the problem of determining its de Rham cohomology was completely solved by

1991 Mathematics Subject Classification: 32C10, 55P62, 53C15.

The paper is in final form and no version of it will be published elsewhere. 
Nomizu [23] in 1954. The Chevalley-Eilenberg complex $\left(\Lambda^{*} \mathfrak{g}^{*}, d\right)$ associated with the Lie algebra $\mathfrak{g}$ of $G$ is canonically identified to the left invariant differential forms on $G$, and then there is a canonical morphism of differential graded algebras

$$
\rho:\left(\Lambda^{*} \mathfrak{g}^{*}, d\right) \longrightarrow\left(\Lambda^{*}(\Gamma \backslash G), d\right),
$$

where $\left(\Lambda^{*}(\Gamma \backslash G), d\right)$ denotes the de Rham complex of $\Gamma \backslash G$. Nomizu proved that $\rho$ induces an isomorphism at the cohomology level. Since the Lie algebra $\mathfrak{g}$ is nilpotent, $\left(\Lambda^{*} \mathfrak{g}^{*}, d\right)$ is a minimal model for the de Rham complex of the nilmanifold $\Gamma \backslash G$.

This result has led to the construction of compact manifolds possessing interesting properties. In fact, since the minimal model $\left(\Lambda^{*} \mathfrak{g}^{*}, d\right)$ is formal if and only if $\mathfrak{g}$ is Abelian [16], a well known result of Deligne, Griffiths, Morgan and Sullivan [12] implies that $\Gamma \backslash G$ has no Kähler structure unless it is a torus (see also [6, 18, 19]). However, many compact nilmanifolds have symplectic structures [7, 28].

In the study of some problems in complex manifold theory it is extremely useful to know a minimal model, in the sense of [22], for the Dolbeault complex of the manifold. In this paper we show such a model for a special class of compact complex manifolds, namely the class of compact nilmanifolds with nilpotent complex structure. As an application we study both the cohomotopy and the cohomology Frölicher spectral sequences for such manifolds.

In order to introduce that special class of complex manifolds, we first remind that on some compact (even dimensional) nilmanifolds $\Gamma \backslash G$ it is possible to define a complex structure in the following way: if the Lie group $G$ has a left invariant complex structure $J$ then $\Gamma \backslash G$ inherits a complex structure by passing $J$ to the quotient. Such a complex manifold will be called a compact complex nilmanifold.

In particular, if $G$ is a complex Lie group then $\Gamma \backslash G$ is a compact complex parallelizable nilmanifold in the sense of Wang [30], that is, there are $n$ holomorphic 1-forms on $\Gamma \backslash G$ ( $n$ being the complex dimension of $G$ ) which are linearly independent at each point.

But, as it is shown in Section 4, some compact complex nilmanifolds admit a complex structure of a special type, which we call nilpotent, that it is not complex parallelizable. In other words, there is an intermediate class of complex manifolds between the class of compact complex nilmanifolds and the class of those which are complex parallelizable. We shall refer to a manifold of this intermediate type as a compact nilmanifold with nilpotent complex structure. In Section 4 we discuss the strictness of the inclusion relations among these three classes of complex nilmanifolds (see Proposition 4.6 and Proposition 4.7).

For the Dolbeault cohomology of a compact complex nilmanifold, a result similar to Nomizu's theorem has been obtained by Sakane [26] only for the particular case of compact complex parallelizable nilmanifolds. In [10] such result has been extended to any compact nilmanifold with nilpotent complex structure $\Gamma \backslash G$ : there is a canonical isomorphism

$$
H_{\bar{\partial}}^{p, q}(\Gamma \backslash G) \cong H_{\bar{\partial}}^{p, q}\left(\mathfrak{g}^{\mathbb{C}}\right),
$$

which reduces the computation of the Dolbeault cohomology of $\Gamma \backslash G$ to calculation at the Lie algebra level $\mathfrak{g}$ of $G$. Moreover, the differential bigraded algebra $\left(\Lambda^{* * *}\left(\mathfrak{g}^{\mathbb{C}}\right), \bar{\partial}\right)$ of complex valued left invariant differential forms on $G$ is a minimal model for the Dolbeault 
complex $\left(\Lambda^{*, *}(\Gamma \backslash G), \bar{\partial}\right)$ of $\Gamma \backslash G$. However, to our knowledge it is still unknown if (1) holds for arbitrary compact complex nilmanifolds.

For complex manifolds $M$, Neisendorfer and Taylor [22] have defined "complex homotopy groups" in terms of the Dolbeault complex of $M$; moreover, the classical Frölicher spectral sequence [14] has a complex homotopy analogue (see Section 3). In Section 4 we compute these complex homotopy invariants for compact nilmanifolds with nilpotent complex structure, and we prove that the cohomotopy spectral sequence is always degenerate for such manifolds.

In contrast, the cohomology Frölicher spectral sequence $\left\{E_{r}\right\}$ of a compact complex parallelizable nilmanifold, not a torus, satisfies $E_{1} \neq E_{2} \cong E_{\infty}$ (see Corollary 4.15). Moreover, there are nilpotent complex structures on compact nilmanifolds for which the sequence $\left\{E_{r}\right\}$ does not collapse even at $E_{2}[8,9,11]$.

In Section 5 we construct a family of compact nilmanifolds with nilpotent complex structure $M_{A B C}$ (depending on three rational parameters $A, B, C$ ), which can be seen as a generalization of the well known Kodaira-Thurston manifold $K T$. More precisely, each $M_{A B C}$ can be described as the total space of a holomorphic principal torus bundle over $K T$. Since $K T$ has complex dimension 2, its associated Frölicher spectral sequence collapses at $E_{1}[4,17]$. However, many complex manifolds $M_{A B C}$ have $E_{2} \neq E_{\infty}$ (see Theorem 5.4). Since $\operatorname{dim}_{\mathbb{C}} M_{A B C}=3$ for any $A, B, C$, then the manifolds $M_{A B C}$ have the lowest possible complex dimension for which one can obtain $E_{2} \nsucceq E_{\infty}$.

The Kodaira-Thurston manifold has no Kähler structure; however, it is symplectic [28] and has indefinite Kähler structures [1]. In Theorem 5.3 we prove that there are complex manifolds $M_{A B C}$ having no indefinite Kähler metric compatible with its complex structure. Even more, some of them have no symplectic structure.

Acknowledgments. This work has been partially supported by DGICYT (Spain), Projects PB94-0633-C02-01 and PB94-0633-C02-02, and by U.P.V. Project 127.310-EC $248 / 96$.

2. Cohomology Frölicher spectral sequence. Let $M$ be a complex manifold. The algebra $\Lambda_{\mathbb{C}}^{*}(M)$ of complex valued differential forms on $M$ admits a natural bigraduation

$$
\Lambda_{\mathbb{C}}^{*}(M)=\bigoplus_{p, q \geq 0} \Lambda^{p, q}(M),
$$

where $\Lambda^{p, q}(M)$ denotes the space of forms of type $(p, q)$ on $M$. The exterior differential $d: \Lambda_{\mathbb{C}}^{*}(M) \longrightarrow \Lambda_{\mathbb{C}}^{*+1}(M)$ decomposes as $d=\partial+\bar{\partial}$, where

$$
\partial: \Lambda^{p, q}(M) \longrightarrow \Lambda^{p+1, q}(M), \quad \bar{\partial}: \Lambda^{p, q}(M) \longrightarrow \Lambda^{p, q+1}(M) .
$$

Since $M$ is a complex manifold, from $d^{2}=0$ it follows that $\partial^{2}=\partial \bar{\partial}+\bar{\partial} \partial=\bar{\partial}^{2}=0$. The complex $\left(\Lambda^{*, *}(M), \bar{\partial}\right)$ is known as the Dolbeault complex of $M$, and its cohomology groups, denoted by $H_{\bar{\partial}}^{p, q}(M)$, are called Dolbeault cohomology groups of $M$.

Since $\partial \bar{\partial}=-\bar{\partial} \partial$ we have also a double complex $\left(\Lambda^{* * *}(M), \partial, \bar{\partial}\right)$ associated with any complex manifold $M$. The Frölicher spectral sequence $\left\{E_{r}(M)\right\}_{r \geq 1}$ of $M$ is the spectral sequence obtained when the double complex $\left(\Lambda^{*, *}(M), \partial, \bar{\partial}\right)$ is derived in vertical directions $\bar{\partial}$. (Notice that one can also derive this double complex in horizontal directions $\partial$; 
however, the terms of the spectral sequence obtained in this way are isomorphic to the terms $E_{r}^{p, q}$ by complex conjugation.) The sequence $\left\{E_{r}(M)\right\}_{r \geq 1}$ satisfies [14]:

$$
E_{1}^{p, q}(M) \cong H_{\bar{\partial}}^{p, q}(M) \text {, }
$$

for any $p, q$, and

$$
\operatorname{Gr} H^{k}(M) \cong \bigoplus_{p+q=k} E_{\infty}^{p, q}(M),
$$

for any $k$, where $H^{*}(M)$ denotes the de Rham cohomology of $M$.

We remark that this sequence can also be seen as the spectral sequence obtained when one considers on $\Lambda_{\mathbb{C}}^{*}(M)$ the decreasing filtration

$$
T^{r}\left(\Lambda_{\mathbb{C}}^{*}(M)\right)=\bigoplus_{p \geq r} \Lambda^{p, *}(M)=\left\{\alpha=\sum \alpha_{p, q} \in \Lambda_{\mathbb{C}}^{*}(M) \mid \alpha_{p, q}=0 \text { for } p<r\right\} .
$$

Notice that, since $d=\partial+\bar{\partial}$, this filtration is compatible with $d$, i.e. $d\left(T^{r}\right) \subset T^{r}$.

The terms $E_{r}^{p, q}(M)$ can be interpreted as quotients $X_{r}^{p, q}(M) / Y_{r}^{p, q}(M)$, where $X_{r}^{p, q}(M)$ in some sense lies between the $\bar{\partial}$-closed and the $d$-closed $(p, q)$-forms, and $Y_{r}^{p, q}(M)$ lies between the $\bar{\partial}$-exact and the $d$-exact $(p, q)$-forms. More precisely:

Theorem 2.1 [9]. Let $M$ be a complex manifold. Then

$$
E_{r}^{p, q}(M) \cong \frac{X_{r}^{p, q}(M)}{Y_{r}^{p, q}(M)}
$$

where

$$
X_{1}^{p, q}(M)=\left\{\alpha \in \Lambda^{p, q}(M) \mid \bar{\partial} \alpha=0\right\}, \quad Y_{1}^{p, q}(M)=\bar{\partial}\left(\Lambda^{p, q-1}(M)\right),
$$

and for $r \geq 2$

$$
\begin{aligned}
& X_{r}^{p, q}(M)=\left\{\alpha_{p, q} \in \Lambda^{p, q}(M) \mid \bar{\partial} \alpha_{p, q}=0\right. \text { and there exist } \\
& \alpha_{p+i, q-i} \in \Lambda^{p+i, q-i}(M) \text { such that } \\
& \left.\partial \alpha_{p+i-1, q-i+1}+\bar{\partial} \alpha_{p+i, q-i}=0, \quad 1 \leq i \leq r-1\right\}, \\
& Y_{r}^{p, q}(M)=\left\{\partial \beta_{p-1, q}+\bar{\partial} \beta_{p, q-1} \in \Lambda^{p, q}(M) \mid\right. \text { there exist } \\
& \beta_{p-i, q+i-1} \in \Lambda^{p-i, q+i-1}(M), 2 \leq i \leq r-1, \\
& \text { satisfying } \quad \partial \beta_{p-i, q+i-1}+\bar{\partial} \beta_{p-i+1, q+i-2}=0 \text {, } \\
& \left.\bar{\partial} \beta_{p-r+1, q+r-2}=0\right\} .
\end{aligned}
$$

Moreover, let $d_{r}: E_{r}^{p, q}(M) \longrightarrow E_{r}^{p+r, q-r+1}(M)$ be the homomorphism given by

$$
d_{r}\left[\alpha_{p, q}\right]=\left[\partial \alpha_{p+r-1, q-r+1}\right],
$$

for $\left[\alpha_{p, q}\right] \in E_{r}^{p, q}(M)$. Then the sequence of homomorphisms

$$
\cdots \longrightarrow E_{r}^{p-r, q+r-1}(M) \stackrel{d_{r}}{\longrightarrow} E_{r}^{p, q}(M) \stackrel{d_{r}}{\longrightarrow} E_{r}^{p+r, q-r+1}(M) \longrightarrow \cdots
$$

satisfies $d_{r}^{2}=0$, and the terms $E_{r+1}^{p, q}(M)$ are isomorphic to the cohomology groups of (5).

From (2) and (3) it follows that the Frölicher spectral sequence relates invariants of the complex structure of $M$ to topological invariants of the manifold. Then, by Hodge theory we have that $E_{1} \cong E_{\infty}$ for any compact Kähler manifold. In [4, 17] it is proved that a compact complex surface has also degenerate Frölicher spectral sequence. 
3. Cohomotopy Frölicher spectral sequence. In this section we outline briefly some results of the Dolbeault homotopy theory developed by Neisendorfer and Taylor in [22]. Roughly speaking this theory is a version of Sullivan's theory for complex manifolds.

In order to introduce the Dolbeault cohomotopy groups of a complex manifold $M$ (of complex dimension $n$ ), we first remind that a $\mathbb{C}$-differential graded algebra, $\mathbb{C}$-DGA, is a commutative graded algebra $\mathcal{A}=\bigoplus_{k \geq 0} \mathcal{A}^{k}$ over $\mathbb{C}$ with a differential $d$ of degree +1 which is a derivation, i.e. $d(a \cdot b)=d a \cdot b+(-1)^{k} a \cdot d b\left(a \in \mathcal{A}^{k}\right)$, and satisfies that $d^{2}=0$.

All the algebras considered here are required to be augmented over $\mathbb{C}$, that is, $(\mathcal{A}, d)$ is endowed with a homomorphism $\alpha: \mathcal{A} \longrightarrow \mathbb{C}$ such that ker $\alpha$ consists of all the elements of positive degree in $\mathcal{A}$.

Definition 3.1. A differential bigraded algebra, DBA, is a $\mathbb{C}$-DGA $(\mathcal{A}, \bar{\partial})$ where the algebra $\mathcal{A}=\bigoplus_{k \geq 0}\left(\bigoplus_{p+q=k} \mathcal{A}^{p, q}\right)$ is bigraded and the differential $\bar{\partial}$ has type $(0,1)$.

We have the category of DBA's by requiring the morphisms between DBA's to be bidegree preserving algebra maps which commute with the differentials $\bar{\partial}$.

A DBA is called a bidifferential bigraded algebra, BBA, if it is equipped with another differential $\partial$ of type $(1,0)$, which is also a derivation and satisfies $\bar{\partial} \partial=-\partial \bar{\partial}$. The cohomology of a $\operatorname{BBA}(\mathcal{A}, \partial, \bar{\partial})$ is defined always with respect to the differential $\bar{\partial}$. Morphisms between BBA's are morphisms between the underlying DBA's which commute with $\partial$.

Let $I(\mathcal{A})$ be the indecomposables of a $\mathbb{C}$-DGA $(\mathrm{DBA}$ or BBA) $\mathcal{A}$, that is, if $A(\mathcal{A})$ denotes the kernel of the augmentation, $I(\mathcal{A})$ is the cokernel of $A(\mathcal{A}) \otimes A(\mathcal{A}) \longrightarrow A(\mathcal{A})$. Then $I(\mathcal{A})$ is a graded (bigraded) vector space with a differential.

ExAmples. Let $M$ be a complex manifold of dimension $n$. As it is shown in Section 2, $\Lambda_{\mathbb{C}}^{*}(M)=\bigoplus_{k \geq 0} \Lambda_{\mathbb{C}}^{k}(M)$, where $\Lambda_{\mathbb{C}}^{k}(M)=\bigoplus_{p+q=k} \Lambda^{p, q}(M)$. Therefore, the de Rham complex $\left(\Lambda_{\mathbb{C}}^{*}(M), d\right)$ is a $\mathbb{C}$-DGA, the Dolbeault complex $\left(\Lambda^{*, *}(M), \bar{\partial}\right)$ is a DBA and the double complex $\left(\Lambda^{* * *}(M), \partial, \bar{\partial}\right)$ is a BBA, where $d=\partial+\bar{\partial}$ is the usual decomposition of the exterior differential $d$ on $M . \Lambda^{*, *}(M)$ is augmented by choosing a point $p$ in $M$ and evaluating the $\mathcal{C}^{\infty}$ complex valued functions on $M$ at $p$.

A DBA $\left(\mathcal{A}, \bar{\partial}_{\mathcal{A}}\right)$ is a model for the Dolbeault complex $\left(\Lambda^{*, *}(M), \bar{\partial}\right)$ of $M$ if there exists a morphism $\rho:\left(\mathcal{A}, \bar{\partial}_{\mathcal{A}}\right) \longrightarrow\left(\Lambda^{*, *}(M), \bar{\partial}\right)$ inducing an isomorphism on cohomology. A model for the de Rham complex $\left(\Lambda_{\mathbb{C}}^{*}(M), d\right)$ of $M$ is defined similarly, but for the double complex $\left(\Lambda^{*, *}(M), \partial, \bar{\partial}\right)$ the morphism $\rho$ is required to be a morphism of BBA's inducing an isomorphism only in $\bar{\partial}$-cohomology.

Instead of Sullivan's minimal models, the authors of [22] use the more functorial version of rational homotopy theory developed by Bousfield and Gugenheim in [5], and introduce the notion of cofibrant model for a DBA. They define a cofibrant DBA as a DBA $\mathcal{A}$ satisfying that: given any surjective morphism of DBA's $g: \mathcal{B} \longrightarrow \mathcal{C}$ which induces an isomorphism on cohomology, and given any morphism $f: \mathcal{A} \longrightarrow \mathcal{C}$, then there exists $h: \mathcal{A} \longrightarrow \mathcal{B}$ such that $g \circ h=f$.

By definition, a cofibrant model for the Dolbeault complex $\left(\Lambda^{*, *}(M), \bar{\partial}\right)$ of a complex manifold $M$ is a model $\mathcal{A}$ which is also a cofibrant DBA. Similar definitions are given for cofibrant models for the de Rham complex and for the double complex of $M$. 
Definition 3.2. The Dolbeault cohomotopy groups of $M$ are the groups $\pi^{*, *}(M)$ defined as follows: if $\mathcal{A}$ is a cofibrant model for the Dolbeault complex of $M$ then $\pi^{*, *}(M)=H^{*, *}(I(\mathcal{A}))$, where $I(\mathcal{A})$ denotes the indecomposables of the DBA $\mathcal{A}$.

REMARK 3.3. In [22] it is proved that $\pi^{*, *}(M)$ is well defined and functorial. Since $\Lambda^{*, *}(M)$ as an augmented algebra depends on the base point $p$, the complex homotopy groups also depend on the choice of $p$. However, if $M$ has no nonconstant holomorphic functions, i.e. $H^{0,0}(M)=\mathbb{C}$, then $\pi^{*, *}(M)$ depends on the base point only up to isomorphism. Since in this paper we deal with compact nilmanifolds, which are connected, this is our case.

The de Rham cohomotopy groups of $M, \pi^{*}(M)$, are the groups $\pi^{*}(M)=H^{*}(I(\mathcal{A}))$, where $I(\mathcal{A})$ denotes the indecomposables of a cofibrant model $\mathcal{A}$ for $\left(\Lambda^{*}(M), d\right)$. For certain complex manifolds $M$ a spectral sequence, similar to the classical Frölicher sequence, relates the Dolbeault cohomotopy of $M$ to the de Rham cohomotopy of the manifold.

TheOREM 3.4 [22]. Let $M$ be a connected complex manifold with no nonconstant holomorphic functions. There exists a spectral sequence $\left\{F_{r}(M)\right\}$ whose term $F_{1}(M)$ is $\left\{\pi^{p, q}(M)\right\}$ and which abuts to $\left\{\pi^{p+q}(M)\right\}$.

Outline of proof. Let $\rho:\left(\mathcal{A}, \partial_{\mathcal{A}}, \bar{\partial}_{\mathcal{A}}\right) \longrightarrow\left(\Lambda^{*, *}(M), \partial, \bar{\partial}\right)$ be a cofibrant model for the double complex of $M$. As in (4), define a filtration on $\mathcal{A}$ by $T^{r}(\mathcal{A})=\oplus_{p \geq r} \mathcal{A}^{p, *}$, which is compatible with the differential $d_{\mathcal{A}}=\partial_{\mathcal{A}}+\bar{\partial}_{\mathcal{A}}$ on $\mathcal{A}$. The morphism $\rho$ maps the spectral sequence for $\mathcal{A}$ to the classical Frölicher spectral sequence. Since $\mathcal{A}$ is also a DBA model (when we forget the differential $\partial_{\mathcal{A}}$ ), this map is an isomorphism on the terms $E_{1}$, and therefore $H^{*}\left(\mathcal{A}, d_{\mathcal{A}}\right)$ is isomorphic to $H^{*}(M)$. This means that $\mathcal{A}$ is a DGA model for $\left(\Lambda^{*}(M), d\right)$.

The filtration $\left\{T^{r}(\mathcal{A})\right\}$ on $\mathcal{A}$ induces a filtration on $I(\mathcal{A})$ compatible with the differential induced by $d_{\mathcal{A}}$. The first term $F_{1}$ of the associated sequence is by Definition 3.2 the Dolbeault cohomotopy of $M$, and since $\left(\mathcal{A}, d_{\mathcal{A}}\right)$ is a model for the de Rham complex $\left(\Lambda^{*}(M), d\right)$ of $M$, then the spectral sequence abuts to the de Rham cohomotopy $H^{*}(I(\mathcal{A}))$.

Finally we remark that the cohomotopy spectral sequence satisfies $F_{1} \cong F_{\infty}$ for any compact Kähler manifold; however, there are complex manifolds for which this sequence does not degenerate at $F_{1}$-level $[22]$.

4. Spectral sequences of compact nilmanifolds with nilpotent complex structure. Let $J$ be a left invariant complex structure on a real $s$-step nilpotent Lie group $G$. Associated with such a complex structure $J$ there exists an ascending series $\left\{\mathfrak{a}_{l}(J)\right\}_{l \geq 0}$ for the Lie algebra $\mathfrak{g}$ of $G$, whose terms $\mathfrak{a}_{l}(J)$ are $J$-invariant ideals in $\mathfrak{g}[10]$. This series is defined inductively as follows:

$\mathfrak{a}_{0}(J)=\{0\}, \quad \mathfrak{a}_{l}(J)=\left\{X \in \mathfrak{g} \mid[X, \mathfrak{g}] \subseteq \mathfrak{a}_{l-1}(J) \quad\right.$ and $\left.[J X, \mathfrak{g}] \subseteq \mathfrak{a}_{l-1}(J)\right\}, \quad l \geq 1$.

We remark that the series $\left\{\mathfrak{a}_{l}(J)\right\}_{l \geq 0}$ depends on the complex structure $J$ considered on the Lie group: in fact, in [10] it is constructed a nilpotent Lie group $G$ admitting two left invariant complex structures $J_{1}$ and $J_{2}$ for which $\mathfrak{a}_{l}\left(J_{1}\right) \neq \mathfrak{a}_{l}\left(J_{2}\right)$ for all $l>0$. 
The ascending series $\left\{\mathfrak{a}_{l}(J)\right\}_{l \geq 0}$ can stop without reaching the Lie algebra $\mathfrak{g}$, that is, it may occur that $\mathfrak{a}_{l}(J)=\mathfrak{a}_{t}(J) \neq \mathfrak{g}$ for all $l \geq t$. This motivates the following:

Definition 4.1. A left invariant complex structure $J$ on $G$ is called nilpotent if there is a positive integer $t$ for which $\mathfrak{a}_{t}(J)=\mathfrak{g}$.

This definition includes the complex structures of complex Lie groups as a particular case.

Proposition 4.2. The canonical complex structure of a complex Lie group is nilpotent.

Proof. Denote by $J$ the canonical complex structure of a complex Lie group $G$. Since the Lie algebra $\mathfrak{g}$ is complex, any ideal of $\mathfrak{g}$ is invariant under $J$ and, in particular, $J\left(\mathfrak{g}_{l}\right)=\mathfrak{g}_{l}$ for $l \geq 0$, where $\left\{\mathfrak{g}_{l}\right\}_{l \geq 0}$ is the usual ascending central series of $\mathfrak{g}$. This implies that $\mathfrak{a}_{l}(J)=\mathfrak{g}_{l}$ for all $l \geq 0$, that is, both series coincide in this case. In particular, if the Lie group is $s$-step nilpotent then $\mathfrak{a}_{s}(J)=\mathfrak{g}_{s}=\mathfrak{g}$ and the complex structure $J$ is nilpotent.

Next we show a characterization of nilpotent complex structures in terms of the structure equations of the Lie group.

Theorem 4.3 [10]. Let $G$ be a nilpotent Lie group of real dimension $2 n$. A left invariant complex structure $J$ on $G$ is nilpotent if and only if there exists an ordered basis $\left\{\omega_{i}, \bar{\omega}_{i} ; 1 \leq i \leq n\right\}$ of left invariant complex 1-forms satisfying

$$
d \omega_{i}=\sum_{j<k<i} A_{i j k} \omega_{j} \wedge \omega_{k}+\sum_{j, k<i} B_{i j k} \omega_{j} \wedge \bar{\omega}_{k}, \quad 1 \leq i \leq n,
$$

where the coefficients $A_{i j k}, B_{i j k}$ are complex numbers. Here each $\omega_{i}$ is of type $(1,0)$ with respect to $J$, and then $\bar{\omega}_{i}$ has type $(0,1)$.

This result allows us to construct in a very simple way nilpotent Lie groups endowed with a nilpotent complex structure. In fact:

COROLlary 4.4. The structure equations (6) with the coefficients chosen so that $d^{2}=$ 0 define a nilpotent Lie group $G$ with a nilpotent left invariant complex structure.

DEFINITION 4.5. A compact nilmanifold with nilpotent complex structure is a complex manifold of the form $\Gamma \backslash G$ whose complex structure is inherited from a nilpotent left invariant complex structure on $G$ by passing to the quotient.

From Proposition 4.2 it follows that the class of compact complex parallelizable nilmanifolds is contained in the class of compact nilmanifolds with nilpotent complex structure. This inclusion is strict for each (even) real dimension $\geq 4$ (in dimension 2 the tori are the only compact nilmanifolds) because the compact complex parallelizable nilmanifolds are precisely those for which all the coefficients $B_{i j k}$ in (6) vanish.

Next we analyse the inclusion relation between the class of compact complex nilmanifolds and the class of those nilmanifolds having a nilpotent complex structure.

First we remind that, apart from tori, the only compact complex nilmanifolds of real dimension $\leq 4$ are Kodaira-Thurston manifolds $K T$ [13]. Moreover, in [2] it is proved 
that for any complex structure on $K T$ coming from a left invariant one, there exists a basis $\left\{\omega_{1}, \omega_{2}, \bar{\omega}_{1}, \bar{\omega}_{2}\right\}$ satisfying

$$
d \omega_{1}=0, \quad d \omega_{2}=\omega_{1} \wedge \bar{\omega}_{1} .
$$

Now Theorem 4.3 implies that the complex structure is nilpotent. Therefore, we have:

Proposition 4.6. In real dimension $\leq 4$, the class of compact complex nilmanifolds coincide with the class of compact nilmanifolds with nilpotent complex structure.

To show that this result does not hold for each even dimension $\geq 6$, let us consider the nilpotent Lie group [3]

$$
G=\left\{\left(\begin{array}{ccccc}
1 & -2 x_{2} & -2 x_{1} & 2\left(x_{1}^{2}+x_{2}^{2}\right) & x_{6} \\
& 1 & 0 & -2 x_{2} & x_{5} \\
& & 1 & -2 x_{1} & x_{4} \\
& & & 1 & x_{3} \\
& & & & 1
\end{array}\right) \mid x_{1}, \ldots, x_{6} \in \mathbb{R}\right\} .
$$

Let $\Gamma$ be the subgroup of $G$ consisting of those matrices whose entries $x_{1}, \ldots, x_{6}$ are integers. Then $L^{6}=\Gamma \backslash G$ is a compact nilmanifold of real dimension 6 .

The functions $x_{1}, \ldots, x_{6}$ are natural coordinates in $G$, and the forms

$d x_{1}, \quad d x_{2}, \quad d x_{3}, \quad d x_{4}+2 x_{1} d x_{3}, \quad d x_{5}+2 x_{2} d x_{3}, \quad d x_{6}+2 x_{2} d x_{5}+2 x_{1} d x_{4}+2\left(x_{1}^{2}+x_{2}^{2}\right) d x_{3}$

constitute a basis for the left invariant 1-forms on $G$. Then, they descend to 1-forms $\alpha_{1}, \ldots, \alpha_{6}$ on $L^{6}$ satisfying

$$
\left\{\begin{aligned}
d \alpha_{1} & =d \alpha_{2}=d \alpha_{3}=0 \\
d \alpha_{4} & =2 \alpha_{1} \wedge \alpha_{3}, \\
d \alpha_{5} & =2 \alpha_{2} \wedge \alpha_{3}, \\
d \alpha_{6} & =2 \alpha_{1} \wedge \alpha_{4}+2 \alpha_{2} \wedge \alpha_{5} .
\end{aligned}\right.
$$

Let $\left\{X_{1}, \ldots, X_{6}\right\}$ be the basis of vector fields on $G$ dual to the basis $\left\{\alpha_{1}, \ldots, \alpha_{6}\right\}$. The left invariant almost complex structure $J$ defined by

$$
J X_{1}=X_{2}, \quad J X_{3}=X_{6}, \quad J X_{4}=X_{5},
$$

is integrable on $G$. A basis $\left\{\omega_{i}, \bar{\omega}_{i} ; 1 \leq i \leq 3\right\}$ for the left invariant complex 1-forms is given by

$$
\omega_{1}=\alpha_{1}+\sqrt{-1} \alpha_{2}, \quad \omega_{2}=\alpha_{3}+\sqrt{-1} \alpha_{6}, \quad \omega_{3}=\alpha_{4}+\sqrt{-1} \alpha_{5} .
$$

In terms of this basis, the structure equations of $G$ are expressed by

$$
\left\{\begin{array}{l}
d \omega_{1}=0 \\
d \omega_{2}=\sqrt{-1}\left(\omega_{1} \wedge \bar{\omega}_{3}-\omega_{3} \wedge \bar{\omega}_{1}\right) \\
d \omega_{3}=\omega_{1} \wedge \omega_{2}+\omega_{1} \wedge \bar{\omega}_{2}
\end{array}\right.
$$

Moreover, there does not exist an ordered basis for the left invariant complex 1-forms on $G$ such that the differential of a generator is expressed in terms of the preceding generators. In fact, it is easy to see that $\mathfrak{a}_{l}(J)=0$ for all $l \geq 0$, and therefore $J$ is nonnilpotent; now Theorem 4.3 implies the nonexistence of such an ordered basis. 
Moreover, since $\mathfrak{a}_{1}(J) \subseteq \mathfrak{g}_{1}=\left\{X_{6}\right\}$ for any left invariant complex structure $J$ on $G$, where $\mathfrak{g}_{1}$ is the center of the Lie algebra of $G$, then $\left\{\mathfrak{a}_{l}(J)\right\}_{l \geq 0}$ always stops at the step $t=0$. Therefore, $L^{6}$ is a compact complex nilmanifold admitting no nilpotent complex structure.

Denote by $\left\{Y_{1}, \ldots, Y_{2 r}\right\}$ a basis for the left invariant vector fields on $\mathbb{R}^{2 r}(r \geq 1)$. Then, the left invariant almost complex structure $J_{r}$ given by

$$
J_{r} X_{1}=X_{2}, \quad J_{r} X_{3}=X_{6}, \quad J_{r} X_{4}=X_{5}, \quad J_{r} Y_{2 i-1}=Y_{2 i},
$$

for $1 \leq i \leq r$, is integrable on the Lie group $G \times \mathbb{R}^{2 r}$. Moreover, the sequence $\left\{\mathfrak{a}_{l}\left(J_{r}\right)\right\}_{l \geq 0}$ satisfies

$$
\mathfrak{a}_{l}\left(J_{r}\right)=\mathfrak{a}_{1}\left(J_{r}\right)=\left\{Y_{1}, \ldots, Y_{2 r}\right\},
$$

for all $l \geq 1$. Since this term does not coincide with the Lie algebra of $G \times \mathbb{R}^{2 r}$, we have that $J_{r}$ is non nilpotent. Therefore, for any $r \geq 1, L^{6} \times \mathbb{T}^{2 r}$ is a compact complex nilmanifold whose complex structure is nonnilpotent. Then, we conclude:

Proposition 4.7. For any (even) real dimension $\geq 6$, the class of compact nilmanifolds with nilpotent complex structure is contained strictly in the class of compact complex nilmanifolds.

4.1. A minimal model for the Dolbeault complex of a compact nilmanifold with nilpotent complex structure. Let $G$ be a nilpotent Lie group with a left invariant almost complex structure $J$. The complexification of the Lie algebra $\mathfrak{g}$ of $G$ can be decomposed as

$$
\mathfrak{g}^{\mathbb{C}}=\mathfrak{g}_{1,0} \oplus \mathfrak{g}_{0,1}
$$

where $\mathfrak{g}_{1,0}=\left\{X \in \mathfrak{g}^{\mathbb{C}} \mid J X=\sqrt{-1} X\right\}$ and $\mathfrak{g}_{0,1}=\left\{X \in \mathfrak{g}^{\mathbb{C}} \mid J X=-\sqrt{-1} X\right\}$. Analogously, $\left(\mathfrak{g}^{*}\right)^{\mathbb{C}}=\mathfrak{g}^{1,0} \oplus \mathfrak{g}^{0,1}$, where $\mathfrak{g}^{1,0}=\left\{\alpha \in\left(\mathfrak{g}^{*}\right)^{\mathbb{C}} \mid J^{*} \alpha=\sqrt{-1} \alpha\right\}$ and $\mathfrak{g}^{0,1}=$ $\left\{\alpha \in\left(\mathfrak{g}^{*}\right)^{\mathbb{C}} \mid J^{*} \alpha=-\sqrt{-1} \alpha\right\}$, where $J^{*} \alpha(X)=\alpha(J X)$. Since $\left(\mathfrak{g}^{\mathbb{C}}\right)^{*} \cong\left(\mathfrak{g}^{*}\right)^{\mathbb{C}}$, there exists a natural bigraduation induced by $J$ on the exterior algebra $\Lambda^{*}\left(\mathfrak{g}^{\mathbb{C}}\right)^{*}$ :

$$
\Lambda^{*}\left(\mathfrak{g}^{\mathbb{C}}\right)^{*}=\bigoplus_{p, q \geq 0} \Lambda^{p, q}\left(\mathfrak{g}^{\mathbb{C}}\right)^{*},
$$

where $\Lambda^{p, q}\left(\mathfrak{g}^{\mathbb{C}}\right)^{*}=\Lambda^{p}\left(\mathfrak{g}^{1,0}\right) \otimes \Lambda^{q}\left(\mathfrak{g}^{0,1}\right)$.

Let us suppose that the almost complex structure $J$ is integrable; then the ChevalleyEilenberg differential $d: \Lambda^{*}\left(\mathfrak{g}^{\mathbb{C}}\right)^{*} \longrightarrow \Lambda^{*+1}\left(\mathfrak{g}^{\mathbb{C}}\right)^{*}$, extended to $\Lambda^{*}\left(\mathfrak{g}^{\mathbb{C}}\right)^{*}$ in a natural way, admits a decomposition as $d=\partial+\bar{\partial}$ where

$$
\partial: \Lambda^{p, q}\left(\mathfrak{g}^{\mathbb{C}}\right)^{*} \longrightarrow \Lambda^{p+1, q}\left(\mathfrak{g}^{\mathbb{C}}\right)^{*}, \quad \bar{\partial}: \Lambda^{p, q}\left(\mathfrak{g}^{\mathbb{C}}\right)^{*} \longrightarrow \Lambda^{p, q+1}\left(\mathfrak{g}^{\mathbb{C}}\right)^{*},
$$

and $\partial^{2}=\partial \bar{\partial}+\bar{\partial} \partial=\bar{\partial}^{2}=0$. Therefore, $\left(\Lambda^{* * *}\left(\mathfrak{g}^{\mathbb{C}}\right)^{*}, \bar{\partial}\right)$ is a DBA.

We remind that a $\operatorname{DBA}\left(\mathcal{A}^{*, *}, \bar{\partial}\right)$ is said to be minimal if $\mathcal{A}^{*, *}$ is free as an algebra and $\bar{\partial}$ is decomposable.

Proposition 4.8. Let $\mathfrak{g}$ be the Lie algebra of a Lie group $G$ endowed with a nilpotent complex structure J. Then $\left(\Lambda^{*, *}\left(\mathfrak{g}^{\mathbb{C}}\right)^{*}, \bar{\partial}\right)$ is a minimal DBA. 
Proof. Since $\Lambda^{* * *}\left(\mathfrak{g}^{\mathbb{C}}\right)^{*}$ is an exterior algebra, it is obviously free. Suppose that $\operatorname{dim}_{\mathbb{R}} G=2 n$ and denote

$$
\Lambda^{*, *}\left(\mathfrak{g}^{\mathbb{C}}\right)^{*} \equiv \Lambda^{*, *}\left(x_{1,0}^{1}, x_{0,1}^{1}, \ldots, x_{1,0}^{n}, x_{0,1}^{n}\right),
$$

where the generators have total degree 1 and bidegree as indexed.

Since $J$ is nilpotent, from Theorem 4.3 it follows the existence of an ordered set of generators $\left\{x_{1,0}^{1}, x_{0,1}^{1}, \ldots, x_{1,0}^{n}, x_{0,1}^{n}\right\}$ with respect to which the differential $\bar{\partial}$ is given by

$$
\bar{\partial} x_{1,0}^{i}=\sum_{j, k<i} B_{i j k} x_{1,0}^{j} \cdot x_{0,1}^{k}, \quad \bar{\partial} x_{0,1}^{i}=\sum_{j<k<i} \bar{A}_{i j k} x_{0,1}^{j} \cdot x_{0,1}^{k},
$$

for $1 \leq i \leq n$. Therefore, $\bar{\partial}$ is decomposable and $\left(\Lambda^{*, *}\left(\mathfrak{g}^{\mathbb{C}}\right)^{*}, \bar{\partial}\right)$ is minimal.

The DBA $\left(\Lambda^{* * *}\left(\mathfrak{g}^{\mathbb{C}}\right)^{*}, \bar{\partial}\right)$ is canonically identified to the DBA of complex valued left invariant forms on the Lie group $G$. Let us suppose that $G$ has a discrete subgroup $\Gamma$ such that $\Gamma \backslash G$ is a compact nilmanifold, and consider on $\Gamma \backslash G$ the complex structure coming from $J$. Since each left invariant form $\alpha$ on $G$ descends to a form on the quotient $\Gamma \backslash G$ whose differential satisfies on $\Gamma \backslash G$ the same relations as $d \alpha$ does on $G$, then there exists a canonical morphism of DBA's

$$
\rho:\left(\Lambda^{*, *}\left(\mathfrak{g}^{\mathbb{C}}\right)^{*}, \bar{\partial}\right) \longrightarrow\left(\Lambda^{*, *}(\Gamma \backslash G), \bar{\partial}\right) .
$$

THEOREM 4.9 [10]. Let $\Gamma \backslash G$ be a compact nilmanifold endowed with a nilpotent complex structure. Then, the morphism (8) induces an isomorphism on cohomology.

From Proposition 4.8 we conclude:

COROLlaRY 4.10. Under the conditions of the above theorem, $\left(\Lambda^{* * *}\left(\mathfrak{g}^{\mathbb{C}}\right)^{*}, \bar{\partial}\right)$ is a minimal model for the Dolbeault complex of $\Gamma \backslash G$.

COROLlary 4.11 (Sakane's Theorem). Let $\Gamma \backslash G$ be a compact complex parallelizable nilmanifold. Then there is a canonical isomorphism

$$
H_{\bar{\partial}}^{p, q}(\Gamma \backslash G) \cong \Lambda^{p}\left(\mathfrak{g}^{1,0}\right) \otimes H^{q}\left(\mathfrak{g}_{0,1}\right),
$$

where $H^{q}\left(\mathfrak{g}_{0,1}\right)$ denotes the cohomology of $\mathfrak{g}_{0,1}$.

Proof. Since the Lie algebra $\mathfrak{g}$ of $G$ is complex, then all the coefficients $B_{i j k}$ in the equations (6) vanish. Then, Proposition 4.8 and Theorem 4.9 imply that a minimal model for the Dolbeault complex of $\Gamma \backslash G$ is the DBA $\left(\Lambda^{* * *}\left(x_{1,0}^{i}, x_{0,1}^{i}\right), 1 \leq i \leq n, \bar{\partial}\right)$ with differential $\bar{\partial}$ given by

$$
\bar{\partial} x_{1,0}^{i}=0, \quad \bar{\partial} x_{0,1}^{i}=\sum_{j<k<i} \bar{A}_{i j k} x_{0,1}^{j} \cdot x_{0,1}^{k},
$$

for $1 \leq i \leq n$. Hence

$$
H_{\bar{\partial}}^{p, q}(\Gamma \backslash G) \cong \Lambda^{p}\left(x_{1,0}^{1}, \ldots, x_{1,0}^{n}\right) \otimes H^{q}\left(\Lambda^{*}\left(x_{0,1}^{1}, \ldots, x_{0,1}^{n}\right)\right),
$$

where $H^{q}\left(\Lambda^{*}\left(x_{0,1}^{1}, \ldots, x_{0,1}^{n}\right)\right)$ denotes the cohomology of the $\operatorname{DGA}\left(\Lambda^{*}\left(x_{0,1}^{1}, \ldots, x_{0,1}^{n}\right), \bar{\partial}\right)$.

4.2. Cohomology Frölicher spectral sequence of compact nilmanifolds with nilpotent complex structure. From now on, $\Gamma \backslash G$ denotes a compact nilmanifold endowed with a nilpotent complex structure $J$. Next we show that the computation of any term $E_{r}^{p, q}(\Gamma \backslash G)$ 
in the cohomology Frölicher spectral sequence of such a complex manifold $\Gamma \backslash G$ is reduced to calculation at the Lie algebra level of $G$.

It is clear that the morphism $\rho$ given by (8) also commutes with the differential $\partial$; therefore, $\rho$ is a morphism between the $\operatorname{BBA}\left(\Lambda^{* * *}\left(\mathfrak{g}^{\mathbb{C}}\right)^{*}, \partial, \bar{\partial}\right)$ and the double complex $\left(\Lambda^{*, *}(\Gamma \backslash G), \partial, \bar{\partial}\right)$ of $\Gamma \backslash G$.

TheOREM 4.12 [11]. In the conditions of Theorem 4.9 , let $\left\{E_{r}\left(\mathfrak{g}^{\mathbb{C}}\right)\right\}_{r \geq 1}$ denote the spectral sequence obtained by derivation of the $B B A\left(\Lambda^{*, *}\left(\mathfrak{g}^{\mathbb{C}}\right)^{*}, \partial, \bar{\partial}\right)$ in vertical direction. Then, for $r \geq 1$ and for any $p, q$, there is a canonical isomorphism

$$
E_{r}^{p, q}(\Gamma \backslash G) \cong E_{r}^{p, q}\left(\mathfrak{g}^{\mathbb{C}}\right) .
$$

Moreover, if $\widetilde{d}_{r}$ denote the homomorphisms corresponding to (5) at the Lie algebra level, then $\left(E_{r}\left(\mathfrak{g}^{\mathbb{C}}\right), \widetilde{d}_{r}\right)$ is a bigraded model for $\left(E_{r}(\Gamma \backslash G), d_{r}\right), r \geq 1$.

As a consequence of this result, in the following proposition we exhibit many examples of compact complex manifolds for which $E_{1} \neq E_{\infty}$.

Proposition 4.13. Let $\Gamma \backslash G$ be a compact complex parallelizable nilmanifold not a complex torus. Then $E_{1}^{1,0}(\Gamma \backslash G) \nRightarrow E_{2}^{1,0}(\Gamma \backslash G)$.

Proof. Since $\Gamma \backslash G$ is complex parallelizable then there are $n$ (holomorphic) 1-forms $\omega_{1}, \ldots, \omega_{n}$ on $\Gamma \backslash G$ satisfying $d \omega_{i}=\sum_{j<k<i} A_{i j k} \omega_{j} \wedge \omega_{k}$, for $1 \leq i \leq n$. Since $\Gamma \backslash G$ is not a torus, then there exists at least one nonzero coefficient $A_{i j k}$. Then the corresponding $\omega_{i}$ is a nonclosed holomorphic 1-form, that is, $\bar{\partial} \omega_{i}=0$ and $\partial \omega_{i} \neq 0$ is a form of type $(2,0)$. From Theorem 4.12 and using the characterization given in Theorem 2.1 for the particular case $(p, q)=(1,0)$, we conclude that $\left[\omega_{i}\right] \neq 0$ in $H_{\bar{\partial}}^{1,0}(\Gamma \backslash G)$, but $\omega_{i}$ does not define a class in $E_{2}^{1,0}(\Gamma \backslash G)$ because $\partial \omega_{i}$ is not $\bar{\partial}$-exact, i.e. $\omega_{i} \notin X_{2}^{1,0}\left(\mathfrak{g}^{\mathbb{C}}\right)$.

The simplest example of a manifold in the conditions of Proposition 4.13 is the well known Iwasawa manifold $I_{3}=\Gamma \backslash H$, defined as the quotient of the complex Heisenberg group by the Gaussian integers. However, this manifold satisfies $E_{2}\left(I_{3}\right) \cong E_{\infty}\left(I_{3}\right)[15]$. More generally:

Proposition 4.14 [8]. Let $\Gamma \backslash G$ be a compact complex parallelizable nilmanifold. Then

for all $p$ and $q$.

$$
E_{2}^{p, q}(\Gamma \backslash G) \cong E_{\infty}^{p, q}(\Gamma \backslash G)
$$

Proof. From Corollary 4.11 we have that

$$
E_{1}^{p, q}(\Gamma \backslash G) \cong \Lambda^{p}\left(\mathfrak{g}^{1,0}\right) \otimes H^{q}\left(\mathfrak{g}_{0,1}\right) .
$$

Now we use (5) at the Lie algebra level. Since $\mathfrak{g}$ is a complex Lie algebra, the homomorphism $\widetilde{d}_{1}=\partial$ maps any element in $H^{q}\left(\mathfrak{g}_{0,1}\right)$ to zero, and $\widetilde{d}_{1}(\alpha)=\partial \alpha=d \alpha$ for any $\alpha \in \Lambda^{p}\left(\mathfrak{g}^{1,0}\right)$. Therefore, from Theorem 4.12 it follows that

$$
E_{2}^{p, q}(\Gamma \backslash G) \cong E_{2}^{p, q}\left(\mathfrak{g}^{\mathbb{C}}\right) \cong H^{p}\left(\mathfrak{g}_{1,0}\right) \otimes H^{q}\left(\mathfrak{g}_{0,1}\right) .
$$

Since for any class $\left[\alpha_{p, q}\right] \in E_{2}^{p, q}\left(\mathfrak{g}^{\mathbb{C}}\right)$ there is a representative satisfying $\bar{\partial} \alpha_{p, q}=\partial \alpha_{p, q}=0$, it is clear that the homomorphism $\widetilde{d}_{r}$ is identically zero for $r \geq 2$, which implies, using 
again Theorem 4.12, that

$$
E_{\infty}^{p, q}(\Gamma \backslash G) \cong E_{\infty}^{p, q}\left(\mathfrak{g}^{\mathbb{C}}\right) \cong E_{2}^{p, q}\left(\mathfrak{g}^{\mathbb{C}}\right) \cong E_{2}^{p, q}(\Gamma \backslash G)
$$

From Proposition 4.13 and Proposition 4.14 we have:

COROLlary 4.15. Let $\Gamma \backslash G$ be a compact complex parallelizable nilmanifold not a complex torus. Then $E_{1}(\Gamma \backslash G) ¥ E_{2}(\Gamma \backslash G) \cong E_{\infty}(\Gamma \backslash G)$.

REMARK 4.16. From the proof in Proposition 4.14 we see that the difference between the terms $E_{1}$ and $E_{2}$ comes from the nonclosed holomorphic forms.

4.3. Cohomotopy Frölicher spectral sequence of compact nilmanifolds with nilpotent complex structure. Let $\left\{F_{r}(M)\right\}$ denote the cohomotopy spectral sequence of a complex manifold $M$ given in Section 3 .

THEOREM 4.17. Let $\Gamma \backslash G$ be a compact nilmanifold endowed with a nilpotent complex structure. Then, the sequence $\left\{F_{r}(\Gamma \backslash G)\right\}_{r \geq 1}$ always collapses at the first level.

Proof. From Theorem 4.9 we have that $\mathcal{A}=\Lambda^{*, *}\left(x_{1,0}^{1}, x_{0,1}^{1}, \ldots, x_{1,0}^{n}, x_{0,1}^{n}\right)$ with differential $\bar{\partial}_{\mathcal{A}}$ given by (7) is a DBA model for the Dolbeault complex $\left(\Lambda^{*, *}(\Gamma \backslash G), \bar{\partial}\right)$ of $\Gamma \backslash G$. Then, the indecomposables $I(\mathcal{A})$ of $\mathcal{A}$ constitute the bigraded vector space generated by $\left\{x_{1,0}^{1}, x_{0,1}^{1}, \ldots, x_{1,0}^{n}, x_{0,1}^{n}\right\}$, and the differential induced by $\bar{\partial}_{\mathcal{A}}$ on $I(\mathcal{A})$ is identically zero. Then $H^{1,0}(I(\mathcal{A}))=\left\langle x_{1,0}^{1}, \ldots, x_{1,0}^{n}\right\rangle, H^{0,1}(I(\mathcal{A}))=\left\langle x_{0,1}^{1}, \ldots, x_{0,1}^{n}\right\rangle$, and $H^{p, q}(I(\mathcal{A}))=\{0\}$ if $p+q \geq 2$.

On the other hand, we know that $\mathcal{A}=\Lambda^{*}\left(x_{1,0}^{1}, x_{0,1}^{1}, \ldots, x_{1,0}^{n}, x_{0,1}^{n}\right)$ with differential $d_{\mathcal{A}}$ given by

$$
\begin{aligned}
& d_{\mathcal{A}}\left(x_{1,0}^{i}\right)=\sum_{j<k<i} A_{i j k} x_{1,0}^{j} \cdot x_{1,0}^{k}+\sum_{j, k<i} B_{i j k} x_{1,0}^{j} \cdot x_{0,1}^{k}, \\
& d_{\mathcal{A}}\left(x_{0,1}^{i}\right)=\sum_{j, k<i} \bar{B}_{i j k} x_{0,1}^{j} \cdot x_{1,0}^{k}+\sum_{j<k<i} \bar{A}_{i j k} x_{0,1}^{j} \cdot x_{0,1}^{k},
\end{aligned}
$$

for $1 \leq i \leq n$, is a DGA model for the de Rham complex of $\Gamma \backslash G$. Then we have that the space of indecomposables $I(\mathcal{A})$ of $\mathcal{A}$ is the graded vector space generated by $x_{1,0}^{1}, x_{0,1}^{1}, \ldots, x_{1,0}^{n}, x_{0,1}^{n}$ (of total degree 1 ). Then the differential on $I(\mathcal{A})$ induced by $d_{\mathcal{A}}$ is identically zero, which implies that $H^{1}(I(\mathcal{A}))=\left\langle x_{1,0}^{1}, x_{0,1}^{1}, \ldots, x_{1,0}^{n}, x_{0,1}^{n}\right\rangle$ and $H^{k}(I(\mathcal{A}))=\{0\}$ for $k \geq 2$.

Since $\left(\mathcal{A}, \bar{\partial}_{\mathcal{A}}\right)$ and $\left(\mathcal{A}, d_{\mathcal{A}}\right)$ are minimal, from Theorem 3.4 and taking into account Definition 3.2, we conclude that the cohomotopy spectral sequence of $\Gamma \backslash G$ satisfies $F_{1}(\Gamma \backslash G) \cong F_{\infty}(\Gamma \backslash G)$.

5. Frölicher spectral sequence in dimension 3. In this section we analyse the behaviour of the cohomology Frölicher spectral sequence $\left\{E_{r}\right\}$ in complex dimension 3, that is the lowest possible dimension in which one can have $E_{1} \neq E_{\infty}[4,17]$. Moreover, in this dimension there exist compact complex manifolds for which the sequence $\left\{E_{r}\right\}$ does not collapse even at $E_{2}$, answering (in the lowest possible dimension) a question posed by Griffiths and Harris in ([15], page 444). 
In view of Corollary 4.15 , to construct nilmanifolds with $E_{2} \not E_{\infty}$ it is necessary to consider at least nilpotent complex structures on compact nilmanifolds. For each triple $(A, B, C)$ of rational numbers, let $G_{A B C}$ be the simply-connected nilpotent Lie group of complex matrices of the form

$$
\left(\begin{array}{ccccccc}
1 & -\frac{A}{2} \bar{z} & -B z & -\frac{B}{2} z^{2} & C \bar{z}-A z & \frac{C}{2} \bar{z}^{2}-A z \bar{z} & w \\
& 1 & 0 & 0 & 0 & 2 z & z^{2} \\
& 1 & z & 0 & 0 & \bar{v} \\
& & 1 & 0 & 0 & \bar{z} \\
& & & 1 & \bar{z} & v \\
& & & & 1 & z \\
& & & & & 1
\end{array}\right)
$$

where $z, v, w \in \mathbb{C}$. (Remark that the Lie group $G_{A B C}$ is not a complex Lie group.) Let $z, v, w: G_{A B C} \longrightarrow \mathbb{C}$ be the natural complex coordinate functions on $G_{A B C}$. Then, an easy computation shows that in terms of $d z, d v, d w$ and their conjugates, a basis for the left invariant complex 1 -forms of type $(1,0)$ on $G_{A B C}$ is given by

$$
\omega_{1}=d z, \quad \omega_{2}=d v-\bar{z} d z, \quad \omega_{3}=d w+A z d v+B z\left(d \bar{v}-\frac{z}{2} d \bar{z}\right)-C \bar{z}\left(d v-\frac{\bar{z}}{2} d z\right) .
$$

Now, with respect to this basis it is clear that the structure equations are:

$$
\left\{\begin{array}{l}
d \omega_{1}=0 \\
d \omega_{2}=\omega_{1} \wedge \bar{\omega}_{1} \\
d \omega_{3}=A \omega_{1} \wedge \omega_{2}+B \omega_{1} \wedge \bar{\omega}_{2}+C \omega_{2} \wedge \bar{\omega}_{1} .
\end{array}\right.
$$

Therefore, from Theorem 4.3 we have that $G_{A B C}$ is a nilpotent Lie group endowed with a nilpotent complex structure for any $(A, B, C) \in \mathbb{Q}^{3}$.

Since $A, B$ and $C$ are rationals, $\left\{\mathfrak{R e}\left(\omega_{i}\right), \mathfrak{I m}\left(\omega_{i}\right) ; 1 \leq i \leq 3\right\}$ constitutes a basis of left invariant real 1-forms on the Lie group such that the coefficients in the structure equations are all rational numbers. Then a well known result by Mal'cev [20] asserts the existence of a lattice $\Gamma_{A B C}$ of $G_{A B C}$ of maximal rank for each $(A, B, C) \in \mathbb{Q}^{3}$. Moreover, we can take $\Gamma_{A B C}$ as the subgroup of $G_{A B C}$ consisting of those matrices (9) whose entries $\{z, v, w\}$ are Gaussian integers. Then $M_{A B C}=\Gamma_{A B C} \backslash G_{A B C}$ is a compact nilmanifold with nilpotent complex structure. Therefore, we have:

Proposition 5.1. The equations (10) define a 3-parametric family of compact nilmanifolds with nilpotent complex structure $M_{A B C}=\Gamma_{A B C} \backslash G_{A B C}$, of complex dimension 3 .

Each $M_{A B C}$ can be seen as a generalization of the well known Kodaira-Thurston manifold $K T$, which is the simplest example of compact nilmanifold with nilpotent complex structure that is real parallelizable but not complex parallelizable. Let $G$ be the simply-connected nilpotent Lie group of complex matrices of the form

$$
\left(\begin{array}{ccc}
1 & \bar{z} & v \\
& 1 & z \\
& & 1
\end{array}\right)
$$


where $z, v \in \mathbb{C}$. In terms of $d z$ and $d v$ we have that

$$
\omega_{1}=d z, \quad \omega_{2}=d v-\bar{z} d z,
$$

constitute a basis for the left invariant $(1,0)$-forms on $G$, with respect to which the structure equations are:

$$
d \omega_{1}=0, \quad d \omega_{2}=\omega_{1} \wedge \bar{\omega}_{1} .
$$

The Kodaira-Thurston manifold $K T$ is the compact nilmanifold with nilpotent complex structure obtained as $K T=\Gamma \backslash G$, where $\Gamma$ is the subgroup of $G$ consisting of those matrices (11) whose entries $\{z, v\}$ are Gaussian integers.

Proposition 5.2. Each $M_{A B C}$ is the total space of a holomorphic principal bundle $\mathbb{T}^{1} \hookrightarrow M_{A B C} \longrightarrow K T$ with structure group the complex torus $\mathbb{T}^{1}$.

Proof. From (9) and (11) it follows that, at the level of Lie groups, $G_{A B C}$ can be described as the total space of a holomorphic principal bundle

$$
\mathbb{C} \hookrightarrow G_{A B C} \stackrel{\pi}{\longrightarrow} G
$$

with Abelian structure group $\mathbb{C}$. In fact, the projection $\pi: G_{A B C} \longrightarrow G$ is given by $\pi(z, v, w)=(z, v)$ and the right action $G_{A B C} \times \mathbb{C} \longrightarrow G_{A B C}$ by $((z, v, w), t) \mapsto(z, v, w+t)$.

Let $\Gamma_{A B C}$ be a lattice of $G_{A B C}$ of maximal rank. Then by passing to the quotient in (12) we get the desired description of $M_{A B C}$.

The Kodaira-Thurston manifold $K T$ was the first known example of a compact symplectic manifold, which is also complex, with no positive definite Kähler metric [7, 17, 28]. Moreover, $K T$ has indefinite Kähler metrics compatible with its natural complex structure [1]. Next we analyse the existence of indefinite Kähler structures, as well as of symplectic structures, on each manifold $M_{A B C}$.

In the particular case $A=B=C=0$, the corresponding complex manifold $M_{000}$ is the product $K T \times \mathbb{T}^{1}$. It is easy to check that

$$
d s^{2}=\omega_{1} \# \bar{\omega}_{2}+\omega_{2} \# \bar{\omega}_{1}+\omega_{3} \# \bar{\omega}_{3},
$$

where \# denotes the symmetric product, is an indefinite Kähler metric compatible with the complex structure of $K T \times \mathbb{T}^{1}$, and the corresponding Kähler form is the symplectic form

$$
F=\sqrt{-1}\left(\omega_{1} \wedge \bar{\omega}_{2}+\omega_{2} \wedge \bar{\omega}_{1}+\omega_{3} \wedge \bar{\omega}_{3}\right) .
$$

Theorem 5.3. Let us suppose $(A, B, C) \in \mathbb{Q}^{3}-\{(0,0,0)\}$.

(i) If $A+B=0$ then, the manifold $M_{A B C}$ has no symplectic structure if and only if $C=0$.

(ii) The complex manifold $M_{A B C}$ has no (compatible) indefinite Kähler metric if and only if $A+B=0$.

Pr o of. To prove (i), let us suppose first that $C \neq 0$. Then $\gamma=\sqrt{-1}\left(-\omega_{1} \wedge \omega_{3}+C \omega_{2} \wedge\right.$ $\left.\bar{\omega}_{2}+\bar{\omega}_{1} \wedge \bar{\omega}_{3}\right)$ is a real closed 2-form satisfying $\gamma^{3}=-6 C \omega_{1} \wedge \omega_{2} \wedge \omega_{3} \wedge \bar{\omega}_{1} \wedge \bar{\omega}_{2} \wedge \bar{\omega}_{3} \neq 0$. Then, it is a symplectic form.

On the other hand, if $A+B=C=0$ then it is easy to see that

$$
H^{2}\left(M_{A B C}\right)=\left\langle\left[\omega_{1} \wedge \omega_{2}\right],\left[\omega_{1} \wedge \omega_{3}\right],\left[\omega_{1} \wedge \bar{\omega}_{3}+\omega_{3} \wedge \bar{\omega}_{1}\right],\left[\bar{\omega}_{1} \wedge \bar{\omega}_{2}\right],\left[\bar{\omega}_{1} \wedge \bar{\omega}_{3}\right]\right\rangle .
$$


Therefore, for any closed 2-form $\gamma$ the class $[\gamma]$ in $H^{2}\left(M_{A B C}\right)$ must be a linear combination of the five classes above. A direct computation shows that $[\gamma]^{3}$ is always zero. Thus, there is no symplectic structure on the manifold.

To prove (ii) we first remark that given a compatible indefinite Kähler structure on a complex manifold $M$, the corresponding Kähler form $F$ is a real closed form of type $(1,1)$ with respect to the complex structure of $M$. Then $F$ defines a class $[F]$ in $H_{\bar{\partial}}^{1,1}(M)$ such that $[F]^{n}$ is a nonzero class in $H_{\bar{\partial}}^{n, n}(M), n$ being the complex dimension of $M$.

Let us suppose that $A+B=0$. From (i) it follows that if $C=0$ then there is no symplectic structure on $M_{A B C}$; therefore, there cannot be an indefinite Kähler metric compatible with the complex structure of $M_{A B C}$. Let us suppose then that the parameter $C \neq 0$. Since the complex structure of $M_{A B C}$ is nilpotent, from the equations (10) and Theorem 4.9 we get

$$
H_{\bar{\partial}}^{1,1}\left(M_{A B C}\right)=\left\langle\left[\omega_{1} \wedge \bar{\omega}_{2}\right],\left[\omega_{1} \wedge \bar{\omega}_{3}+\omega_{3} \wedge \bar{\omega}_{1}\right],\left[\omega_{1} \wedge \bar{\omega}_{3}+A \omega_{2} \wedge \bar{\omega}_{2}\right]\right\rangle .
$$

Then, for any cohomology class $[F]$ in $H_{\bar{\partial}}^{1,1}\left(M_{A B C}\right)$ there are complex numbers $\lambda, \mu, \nu$ such that

$$
[F]=\lambda\left[\omega_{1} \wedge \bar{\omega}_{2}\right]+\mu\left[\omega_{1} \wedge \bar{\omega}_{3}+\omega_{3} \wedge \bar{\omega}_{1}\right]+\nu\left[\omega_{1} \wedge \bar{\omega}_{3}+A \omega_{2} \wedge \bar{\omega}_{2}\right] .
$$

If $F$ is the corresponding Kähler form of a compatible indefinite Kähler metric on $M_{A B C}$ then $F=\bar{F}$, which implies that $[F]-[\bar{F}]$ is zero in $H_{\bar{\partial}}^{1,1}\left(M_{A B C}\right)$. From (13) we have

$$
\begin{aligned}
{[F]-[\bar{F}]=[} & \lambda \omega_{1} \wedge \bar{\omega}_{2}+\bar{\lambda} \omega_{2} \wedge \bar{\omega}_{1}+(\mu+\bar{\mu}+\nu) \omega_{1} \wedge \bar{\omega}_{3} \\
& \left.+(\mu+\bar{\mu}+\bar{\nu}) \omega_{3} \wedge \bar{\omega}_{1}+A(\nu+\bar{\nu}) \omega_{2} \wedge \bar{\omega}_{2}\right] .
\end{aligned}
$$

Denote by $\alpha$ the $(1,1)$-form on the right hand side of (14). Since $\alpha \in \Lambda^{1,1}\left(\mathfrak{g}_{A B C}^{\mathbb{C}}\right)^{*}, \mathfrak{g}_{A B C}$ being the Lie algebra of $G_{A B C}$, from Theorem 4.9 we have that $[F]-[\bar{F}]$ is zero in $H_{\bar{\partial}}^{1,1}\left(M_{A B C}\right)$ if and only if $\alpha \in \bar{\partial}\left(\Lambda^{1,0}\left(\mathfrak{g}_{A B C}^{\mathbb{C}}\right)^{*}\right)$. But from equations (10) for $B=-A$ we have that $\bar{\partial}\left(\Lambda^{1,0}\left(\mathfrak{g}_{A B C}^{\mathbb{C}}\right)^{*}\right)=\left\langle\omega_{1} \wedge \bar{\omega}_{1},-A \omega_{1} \wedge \bar{\omega}_{2}+C \omega_{2} \wedge \bar{\omega}_{1}\right\rangle$. Therefore, we conclude that $F=\bar{F}$ implies in particular that $\lambda, \mu$ and $\nu$ must satisfy

$$
\mu+\bar{\mu}+\nu=\mu+\bar{\mu}+\bar{\nu}=A(\nu+\bar{\nu})=0 .
$$

On the other hand, a simple calculation using (13) shows that

$$
[F]^{3}=\left[6 \mu \nu(\mu+\nu) A \omega_{1} \wedge \omega_{2} \wedge \omega_{3} \wedge \bar{\omega}_{1} \wedge \bar{\omega}_{2} \wedge \bar{\omega}_{3}\right]
$$

Therefore, if $A=0$ then $[F]^{3}=0$. Let us suppose $A \neq 0$; then from equations (15) it follows that $\nu=0$, which implies again $[F]^{3}=0$. Thus, $[F]^{3}$ is always zero if $A+B=0$, that is, there is no compatible indefinite Kähler metric on the complex manifolds $M_{A B C}$ with $A+B=0$.

To prove the reciprocity, let us suppose that $A+B \neq 0$. Then,

$$
d s^{2}=\omega_{1} \# \bar{\omega}_{3}+(A+B) \omega_{2} \# \bar{\omega}_{2}+\omega_{3} \# \bar{\omega}_{1}
$$

is an indefinite Kähler metric on $M_{A B C}$ and the corresponding Kähler form is the symplectic form $F=\sqrt{-1}\left(\omega_{1} \wedge \bar{\omega}_{3}+(A+B) \omega_{2} \wedge \bar{\omega}_{2}+\omega_{3} \wedge \bar{\omega}_{1}\right)$.

Since $K T$ has complex dimension 2, its associated Frölicher spectral sequence collapses at $E_{1}$. However, there are many complex manifolds $M_{A B C}$ even with $E_{2} \not E_{\infty}$. In fact, 
in the following theorem we exhibit the behaviour of the Frölicher spectral sequence for each manifold $M_{A B C}$.

TheOREM 5.4. Let $M_{A B C}$ be a compact complex manifold defined by (10). Then,

$$
E_{2}\left(M_{A B C}\right) \nRightarrow E_{\infty}\left(M_{A B C}\right) \text { if and only if } C \neq 0 \text { and } C^{2} \neq(B-A)^{2} \text {. }
$$

Moreover, if $E_{2}\left(M_{A B C}\right) \approx E_{\infty}\left(M_{A B C}\right)$ then

$$
E_{1}\left(M_{A B C}\right) \nRightarrow E_{2}\left(M_{A B C}\right) \quad \text { if and only if } \quad A=0 \text {. }
$$

We omit here the proof of this result, which reduces to a long, but easy calculation using Theorem 4.12 (in [9, 11] a detailed proof of Theorem 5.4 can be found). However, we remark that the algebraic conditions appearing in Theorem 5.4 have a topological meaning. In fact, if $C \neq 0$ and $C^{2} \neq(B-A)^{2}$ then it is easy to prove that $H^{1}\left(M_{A B C}\right)=$ $\left\langle\left[\omega_{1}\right],\left[\bar{\omega}_{1}\right],\left[\omega_{2}+\bar{\omega}_{2}\right]\right\rangle$, that is, $M_{A B C}$ has first Betti number $b_{1}\left(M_{A B C}\right)=3$, which is the lowest possible $b_{1}$ for a nilmanifold defined by (10). This implies that the dimension of the de Rham cohomology $E_{\infty}\left(M_{A B C}\right)$ of $M_{A B C}$ is small, which provides an explanation of the behaviour of the sequence $\left\{E_{r}\right\}$ for such manifolds.

Moreover, if the relations $C \neq 0$ and $C^{2} \neq(B-A)^{2}$ are satisfied then, $A=0$ if and only if $\bar{\omega}_{3}$ is a $\bar{\partial}$-closed form, which is equivalent to say that the Hodge number $h_{0,1}\left(M_{A B C}\right)=\operatorname{dim} H_{\bar{\partial}}^{0,1}\left(M_{A B C}\right)$ is maximum (i.e. $h_{0,1}\left(M_{A B C}\right)=3$ ). This implies that the dimension of the Dolbeault cohomology $E_{1}\left(M_{A B C}\right)$ of $M_{A B C}$ is large, which explains that $E_{1} \approx E_{2} \approx E_{\infty}$ for such manifolds.

In the following table we illustrate the dimension of each term in the sequence

\begin{tabular}{|c|c|c|c|c|c|}
\hline \multicolumn{2}{|c|}{ parameters $A, B, C$} & $\operatorname{dim} E_{1}$ & $\operatorname{dim} E_{2}$ & $\operatorname{dim} E_{3}$ & sequence $\left\{E_{r}\right\}$ \\
\hline \multicolumn{2}{|c|}{$A=0 ; C \neq 0, \pm B$} & 32 & 28 & 24 & $E_{1} \nsucceq E_{2} \nsucceq E_{3} \cong E_{\infty}$ \\
\hline \multicolumn{2}{|c|}{$A \neq 0 ; B \neq 0, A ; C=0$} & 36 & 24 & 24 & $E_{1} \nsucceq E_{2} \cong E_{\infty}$ \\
\hline \multirow{2}{*}{$A, B, C \neq 0$} & $C^{2} \neq(B-A)^{2}$ & 28 & 28 & 24 & $E_{1} \cong E_{2} \nsucceq E_{3} \cong E_{\infty}$ \\
\hline & $C^{2}=(B-A)^{2}$ & 28 & 28 & 28 & $E_{1} \cong E_{\infty}$ \\
\hline
\end{tabular}
$\left\{E_{r}\left(M_{A B C}\right)\right\}_{r \geq 1}$, for some particular manifolds $M_{A B C}$ in the family defined by (10):

From Theorem 5.3 we have:

(i) If $A+B=0$ in the above table, then $M_{A B C}$ belongs to the class of compact complex manifolds with no (compatible) indefinite Kähler metric.

(ii) If $A+B \neq 0$ in the above table, then $M_{A B C}$ belongs to the class of compact indefinite Kähler manifolds.

As we see in the table, in these two special subclasses of compact complex manifolds one can find nilmanifolds $M_{A B C}$ for which the sequence $\left\{E_{r}\right\}$ satisfies any of the four possible behaviours in dimension 3 . In this sense, we conclude that (in complex dimension 3) the nonexistence, as well as the existence, of a compatible indefinite Kähler metric (not 
positive definite, of course) on the complex manifold has no influence on the behaviour of the Frölicher spectral sequence.

\section{References}

[1] L. C. de Andrés, M. Fernández, A. Gray and J. J. Mencía, Compact manifolds with indefinite Kähler metrics, Proc. VIth Int. Coll. Differential Geometry (Ed. L.A. Cordero), Santiago (Spain) 1988, Cursos y Congresos 61, 25-50, Univ. Santiago de Compostela (Spain) 1989.

[2] L. C. de Andrés, M. Fernández, A. Gray and J. J. Mencía, Moduli spaces of complex structures on compact four dimensional nilmanifolds, Bolletino U.M.I. (7) 5-A (1991), 381-389.

[3] E. Abbena, S. Garbiero and S. Salamon, private communication.

[4] W. Barth, C. Peters and A. Van de Ven, Compact Complex Surfaces, Ergebnisse der Mathematik (3) 4, Springer-Verlag, Berlin, Heidelberg, 1984.

[5] A. K. Bousfield and V. K. A. M. Gugenheim, On PL de Rham theory and rational homotopy type, Memoirs Amer. Math. Soc. 8 (179) (1976).

[6] C. Benson and C. Gordon, Kähler and symplectic structures on nilmanifolds, Topology 27 (1988), 513-518.

[7] L. A. Cordero, M. Fernández and A. Gray, Symplectic manifolds with no Kähler structure, Topology 25 (1986), 375-380.

[8] L. A. Cordero, M. Fernández and A. Gray, The Frölicher spectral sequence for compact nilmanifolds, Illinois J. Math. 35 (1991), 56-67.

[9] L. A. Cordero, M. Fernández, A. Gray and L. Ugarte, A general description of the terms in the Frölicher spectral sequence, Diff. Geom. Appl. 7 (1997), 75-84.

[10] L. A. Cordero, M. Fernández, A. Gray and L. Ugarte, Compact nilmanifolds with nilpotent complex structure: Dolbeault cohomology, preprint 1997.

[11] L. A. Cordero, M. Fernández, A. Gray and L. Ugarte, Frölicher spectral sequence of compact nilmanifolds with nilpotent complex structure, Proc. Conference on Differential Geometry, Budapest, July 27-30, 1996 (to appear).

[12] P. Deligne, P. Griffiths, J. Morgan and D. Sullivan, Real homotopy theory of Kähler manifolds, Invent. Math. 29 (1975), 245-274.

[13] M. Fernández, M. J. Gotay and A. Gray, Four-dimensional compact parallelizable symplectic and complex manifolds, Proc. Amer. Math. Soc. 103 (1988), 1209-1212.

[14] A. Frölicher, Relations between the cohomology groups of Dolbeault and topological invariants, Proc. Nat. Acad. Sci. U.S.A. 41 (1955), 641-644.

[15] P. Griffiths and J. Harris, Principles of Algebraic Geometry, Wiley, New York, 1978.

[16] K. Hasegawa, Minimal models of nilmanifolds, Proc. Amer. Math. Soc. 106 (1989), $65-71$.

[17] K. Kodaira, On the structure of compact complex analytic surfaces, I, Amer. J. Math. 86 (1964), 751-798.

[18] G. Lupton and J. Oprea, Symplectic manifolds and formality, J. Pure Appl. Algebra 91 (1994), 193-207.

[19] G. Lupton and J. Oprea, Cohomologically symplectic spaces: toral actions and the Gottlieb group, Trans. Amer. Math. Soc. 347 (1995), 261-288.

[20] I. A. Mal'cev, A class of homogeneous spaces, Amer. Math. Soc. Transl. No. 39 (1951). 
[21] I. Nakamura, Complex parallelisable manifolds and their small deformations, J. Differential Geom. 10 (1975), 85-112.

[22] J. Neisendorfer and L. Taylor, Dolbeault Homotopy Theory, Trans. Amer. Math. Soc. 245 (1978), 183-210.

[23] K. Nomizu, On the cohomology of compact homogeneous spaces of nilpotent Lie groups, Ann. of Math. 59 (1954), 531-538.

[24] J. Oprea and A. Tralle, Symplectic Manifolds with no Kähler Structure, Lecture Notes in Math. 1661, Springer, Berlin, 1997.

[25] H. Pittie, The nondegeneration of the Hodge-de Rham spectral sequence, Bull. Amer. Math. Soc. 20 (1989), 19-22.

[26] Y. Sakane, On compact parallelisable solvmanifolds, Osaka J. Math. 13 (1976), 187-212.

[27] D. Tanré, Modèle de Dolbeault et fibré holomorphe, J. Pure Appl. Algebra 91 (1994), 333-345.

[28] W. P. Thurston, Some simple examples of symplectic manifolds, Proc. Amer. Math. Soc. 55 (1976), 467-468.

[29] A. Tralle, Applications of rational homotopy to geometry (results, problems, conjectures), Expo. Math. 14 (1996), 425-472.

[30] H. C. Wang, Complex parallelisable manifolds, Proc. Amer. Math. Soc. 5 (1954), 771-776. 